\title{
Cavernous hemangioma of the skull presenting with subdural hematoma
}

\author{
Case report
}

\author{
Oren N. Gottfried, M.D., Wayne M. Gluf, M.D., And Meic H. Schmidt, M.D. \\ Department of Neurosurgery, University of Utah, Salt Lake City, Utah
}

\begin{abstract}
Cavernous hemangioma of the calvaria is a very rare disease, and patients usually present with headaches or a visible skull deformity. Few reports of patients presenting with intradiploic or epidural hemorrhages are found in the literature. No case of an intradural hemorrhage from a cavernous hemangioma of the skull has been reported to date. The authors present the case of a 50-year-old man in whom a symptomatic subdural hematoma (SDH) resulting from a cavernous hemangioma of the calvaria had hemorrhaged and eroded through the inner table of the skull and dura mater. The patient underwent surgery for evacuation of the SDH and resection of the calvarial lesion. Postoperatively, the patient experienced immediate relief of his symptoms and had no clinical or radiological recurrence. Calvarial cavernous hemangiomas should be considered in the differential diagnosis of nontraumatic SDHs. Additionally, skull lesions that present with intracranial hemorrhages must be identified and resected at the time of hematoma evacuation to prevent recurrences.
\end{abstract}

KEY WORDS - calvaria - cavernous hemangioma - subdural hematoma

Cavernous hemangioma of the skull is a rare pathological diagnosis, accounting for $0.2 \%$ of benign skull lesions. ${ }^{2}$ Cavernous hemangiomas are commonly found in patients who are in the middle decades of life, and the peak incidence occurs in the fourth or fifth decade. ${ }^{6}$ These lesions grow between the outer and inner tables of the skull, are supplied by the branches of the external carotid artery in the diploic space, are usually solitary, and typically occur in the frontal or parietal bones. ${ }^{2,46}$ In a literature review of cavernous hemangiomas of the skull, Heckl, et al., ${ }^{4}$ found that the lesion could occur in almost any skull region, including (in descending order of incidence) the frontal, temporal, parietal, mandibular, zygomatic, nasal, maxillary, occipital, sphenoidal, clival, and ethmoidal regions. Typically, cavernous hemangiomas grow slowly before they cause symptoms of pain or a visible or palpable skull deformity; however, they can cause a variety of symptoms depending on the location of the lesion. ${ }^{6,8,9}$ Few reports of cavernous hemangiomas presenting with intradiploic or epidural hemorrhages have appeared. ${ }^{5,8,9}$ We present the unique case of a patient with a calvarial cavernous hemangioma that resulted in a large subdural hemorrhage. We also review the literature on patients with cavernous hemangiomas of the skull who present with hemorrhage.

Abbreviations used in this paper: $\mathrm{CT}=$ computerized tomography; $\mathrm{MR}=$ magnetic resonance; $\mathrm{SDH}=$ subdural hematoma.

\section{CASE REPORT}

History and Examination. This 50-year-old man presented to our hospital after 1 week of increasing headaches, nausea, left arm weakness with poor coordination, and difficulty walking because of a "clumsy" left leg. His physical examination was significant for mild proximal weakness in both the left arm and leg, with marked pronator drift of the left upper extremity. The patient's prothrombin time, partial thromboplastin time, and international normalized ratio were in the normal range.

Neuroimaging. On CT scans, an extraaxial, frontoparietal, hypodense fluid collection was visible on the right side; this was identified as a chronic SDH (Fig. 1B). This fluid collection was associated with an expanding calvarial lesion located in the right high parietal bone that had eroded through the inner table of the skull and had distinct margins with associated sclerosis (Fig. 1A and B). On MR imaging, a large right-sided SDH with late subacute signal features and evidence of a more acute superimposed hemorrhage were apparent. Numerous internal locules were present, indicating the typical evolution of a large, organized hematoma. We also found a right-sided parietal expanding lesion measuring $3.5 \times 1.3 \mathrm{~cm}$ in the diploic space; the lesion was hyperintense on both $\mathrm{T}_{1^{-}}$and $\mathrm{T}_{2^{-}}$ weighted sequences and enhanced after administration of Gd contrast material (Fig. 1C and D). The calvarial lesion had single-scalloped cortical margins and a heterogeneous 


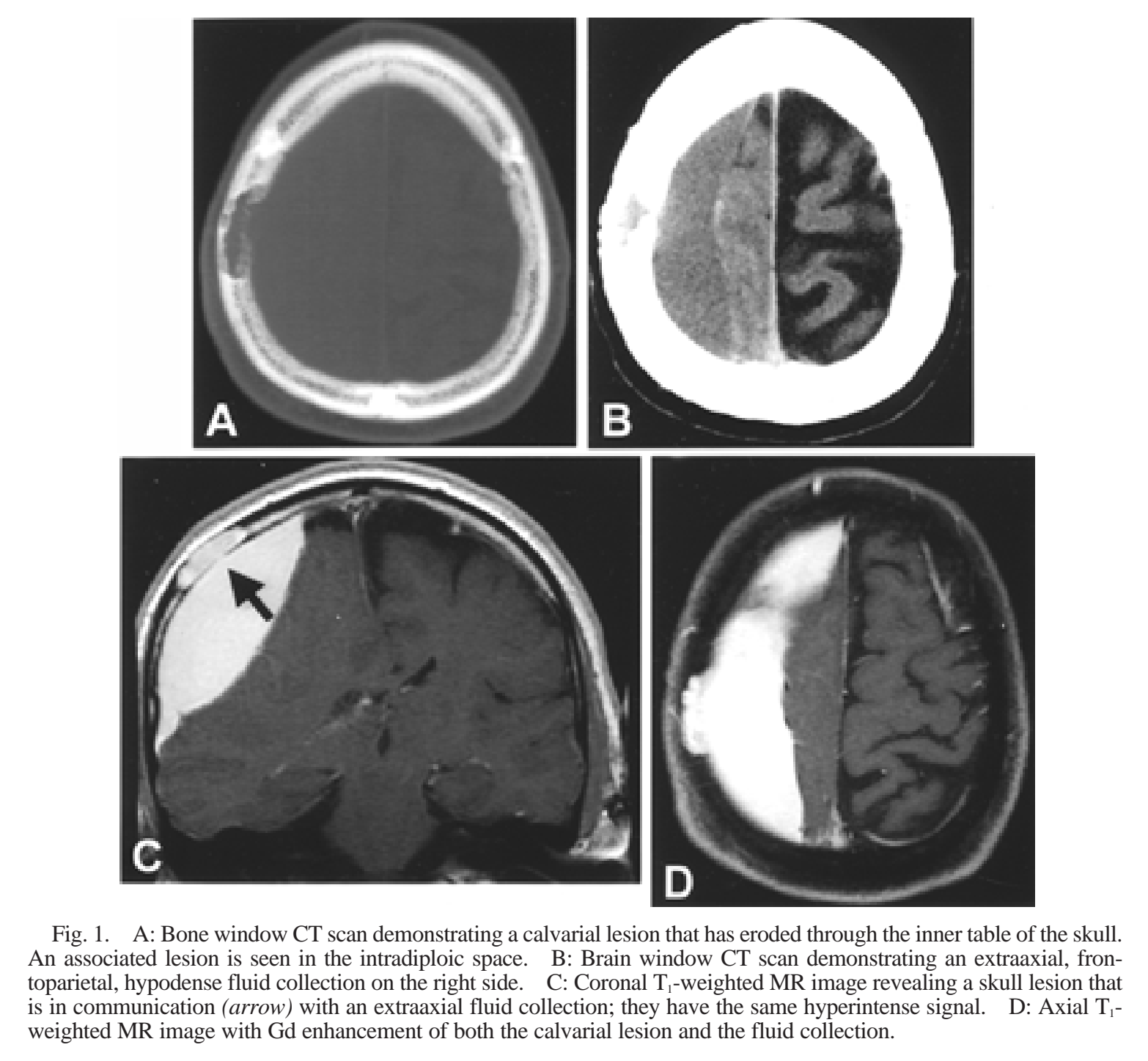

internal signal, and it followed the course of the SDH signal on all MR imaging sequences. Figure $1 \mathrm{C}$ demonstrates a communication between the lesion and the SDH, which indicates that a dural perforation was present and that the skull lesion resulted in the SDH.

Operation. The patient was brought to the operating room for evacuation of the SDH and resection of the calvarial lesion. Dark stippling of the bone surface was obvious on elevation of the myocutaneous skin flap (Fig. 2A). A 3-cm craniotomy centered on the skull lesion was performed, and the lesion was excised to clean margins. Complete erosion through the inner table had occurred (Fig. 2B). Additionally, the dura mater was eroded all the way through in the area immediately subjacent to the skull cavernous hemangioma, making the subdural space contiguous with the calvarial lesion (Fig. 2C). Although Fig. $1 \mathrm{C}$ indicated that the hematoma was located in the epidural space, at surgery we confirmed the presence of an $\mathrm{SDH}$. Fluid resembling motor oil drained under considerable pressure after removal of the bone flap (Fig. 2C). The dura mater was opened completely and the SDH was evacuated. Subdural membranes indicated the acute-plus- chronic nature of the hemorrhage, and the membranes were not resected. Cranioplasty was performed and a subdural drain was left in place for 24 hours.

Postoperative Course. Postoperatively, the patient noted immediate relief of symptoms, and results of his neurological examination were normal, with complete recovery from his motor weakness. He was discharged home on postoperative Day 3 after an uneventful course. Follow-up images obtained immediately postoperatively and at later intervals demonstrated resolution of the SDH.

\section{Pathological Findings}

Macroscopically, the bone displayed a purple-red blush or discoloration in an area measuring $3.5 \times 3 \mathrm{~cm}$ on the outer surface. The inner table of the calvaria was completely eroded centrally over an area measuring $1.2 \times 1.1$ $\mathrm{cm}$. The defect was surrounded by a purple blush associated with multiple, small, confluent pits. The inner table was markedly thinned around the large central defect for approximately $1 \mathrm{~cm}$. The margins of the bone were free of the discoloration. Microscopic examination revealed blood-filled sinusoidal channels lined with a single 
layer of flattened endothelial cells mixed with bone trabeculae (Fig. 2D). The histological diagnosis was of a cavernous hemangioma.

\section{DISCUSSION}

Cavernous hemangioma of the skull is a rare pathological diagnosis. ${ }^{2}$ Typically, cavernous hemangiomas grow slowly before they cause symptoms, which include pain or a visible or palpable skull deformity. These lesions can cause a variety of symptoms depending on their location. ${ }^{6,8,9}$ We present the unique case of a patient with a calvarial cavernous hemangioma that resulted in a subdural hemorrhage.

Although most patients with cavernous hemangiomas present with pain or local skull deformities, there have been a few reports in the literature of epidural or intradiploic hemorrhages occurring spontaneously or after minimal trauma. ${ }^{5,8,9}$ Cavernous hemangiomas usually erode the outer table and are confined by the inner table, and, despite their enlargement and subsequent erosion of the surrounding bone, the inner table remains intact. $4,6,8,9$ Uemura, et al., ${ }^{9}$ described a patient with a calvarial cav- ernous hemangioma who presented with an epidural hemorrhage in which the inner table was completely eroded and the lesion was in direct contact with intact dura mater. It is hypothesized that the epidural hematomas arising from cavernous hemangiomas occur because of fragile vessels contacting the dura mater through the pores of the inner table or from minor trauma, causing fracture of an already thinned inner table. ${ }^{8,9}$ Interestingly, isolated cases of extraaxial hematomas arising from other skull lesions, including Ewing sarcoma, ${ }^{10}$ an epidermoid lesion, ${ }^{1}$ a hemangioendothelioma, ${ }^{7}$ and metastasis from hepatocellular carcinoma ${ }^{3}$ have been reported, but in these cases the dura mater remained intact, leading to epidural hemorrhage.

Unlike previous cases of cavernous hemangiomas and other skull lesions in which the hemorrhage was contained by an intact dura, in the current case the lesion eroded through the dura mater. It is possible that the pressure from an intradiploic hemorrhage resulted in the perforation of the dura mater and remaining inner table, resulting in the SDH. Also uniquely, the patient had no history of recent or remote trauma, indicating a more gradual process. Blood products and numerous subdural membranes of various ages indicated that there had been hemorrhage

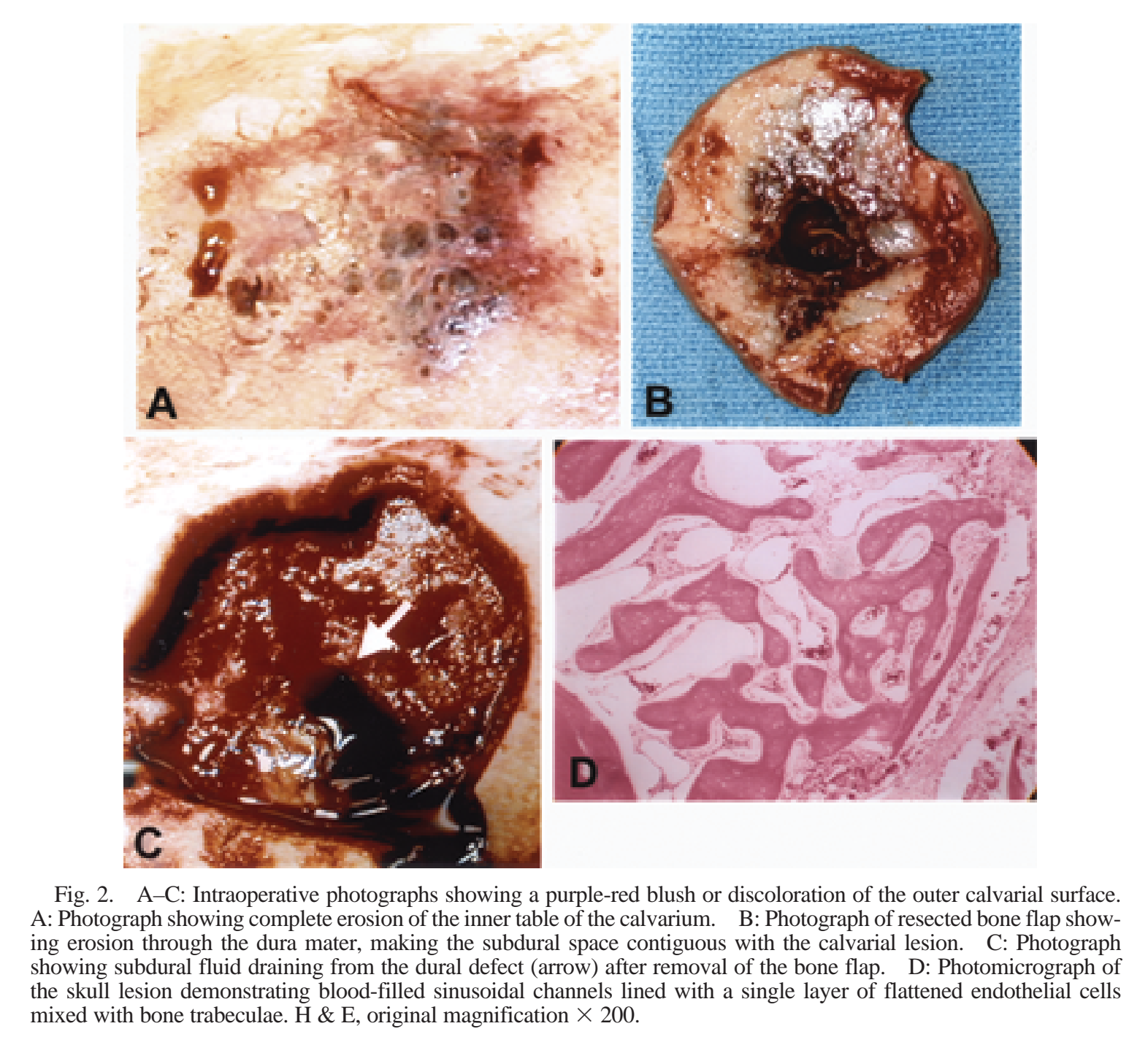


into the subdural space on several occasions. We hypothesize that multiple hemorrhages over time weakened the dura mater, leading to its erosion.

Overall, a cavernous hemangioma of the calvaria should be considered in the differential diagnosis of a nontraumatic SDH. If a patient presents with an extraaxial hemorrhage and has an associated skull lesion, resection of the skull lesion to clean margins at the time of evacuation of the hematoma is necessary. If the fluid collection is evacuated without simultaneous resection of the calvarial mass, the patient may experience subsequent hemorrhages and symptomatic events.

\section{References}

1. Abou-Samra M, Marlin AE, Story JL, et al: Cranial epidermoid tumor associated with subacute extradural hematoma. Case report. J Neurosurg 53:574-575, 1980

2. Barnes L: Solitary hemangiomas of bone, in Barnes L (ed): Surgical Pathology of the Head and Neck. New York: Dekker, 1985, pp 932-936

3. Hayashi K, Matsuo T, Kurihara M, et al: Skull metastasis of hepatocellular carcinoma associated with acute epidural hematoma: a case report. Surg Neurol 53:379-382, 2000

4. Heckl S, Aschoff A, Kunze S: Cavernomas of the skull: review of the literature 1975-2000. Neurosurg Rev 25:56-62, 66-67, 2002

5. Kessler LA, Lubic LG, Koskoff YD: Epidural hemorrhage secondary to cavernous hemangioma of the petrous portion of the temporal bone. J Neurosurg 14:329-331, 1957

6. Khanam H, Lipper MH, Wolff CL, et al: Calvarial hemangiomas: report of two cases and review of the literature. Surg Neurol 55:63-67, 2001

7. Maroon JC, Haines SJ, Phillips JG: Calvarial hemangioendothelioma with intracranial hemorrhage: case report. Neurosurgery 4:178-180, 1979

8. Naim-Ur-Rahman, Hafeez MA: Haemorrhage in intradiploic haemangioma mimicking extradural haematoma. Neuroradiology 33:529-531, 1991

9. Uemura K, Takahashi S, Sonobe M, et al: Intradiploic haemangioma associated with epidural haematoma. Neuroradiology 38:456-457, 1996

10. Yamashita Y, Kumabe T, Kobayashi T, et al: [Ewing's sarcoma at the occipital bone presenting as acute epidural hematoma: case report.] No Shinkei Geka 25:567-571, 1997 (Jpn)

Manuscript received June 29, 2004.

Accepted in final form July 15, 2004.

Address reprint requests to: Meic H. Schmidt, M.D., Department of Neurosurgery, University of Utah Medical Center, 30 North 1900 East, Suite 3B409, Salt Lake City, Utah 84132. email: Meic.Schmidt@hsc.utah.edu. 